CERN-PH-TH/2007-196

\title{
Exploiting symmetries for exponential error reduction in path integral Monte Carlo
}

\author{
Michele Della Morte and Leonardo Giusti
}

CERN, Physics Department, TH Division, CH-1211 Geneva 23, Switzerland

\begin{abstract}
The path integral of a quantum system with an exact symmetry can be written as a sum of functional integrals each giving the contribution from quantum states with definite symmetry properties. We propose a strategy to compute each of them, normalized to the one with vacuum quantum numbers, by a Monte Carlo procedure whose cost increases power-like with the time extent of the lattice. This is achieved thanks to a multi-level integration scheme, inspired by the transfer matrix formalism, which exploits the symmetry and the locality in time of the underlying statistical system. As a result the cost of computing the lowest energy level in a given channel, its multiplicity and its matrix elements is exponentially reduced with respect to the standard path-integral Monte Carlo. We test the strategy with a one-dimensional harmonic oscillator, by computing the ratio of the parity odd over the parity even functional integrals and the two-point correlation function. The cost of the simulations scales as expected. In particular the effort for computing the lowest energy eigenvalue in the parity odd sector grows linearly with the time extent. At a fixed CPU time, the statistical error on the two-point correlation function is exponentially reduced with respect to the standard Monte Carlo procedure, and at large time distances it is lowered by many orders of magnitude.
\end{abstract}




\section{Introduction}

The path integral formulation allows to study dynamical properties of quantum systems by Monte Carlo simulations. For many quantum field theories, for instance, it is the only tool to compute time-ordered products of operators from first principles non-perturbatively.

The lowest energy eigenvalue in a given channel can, for instance, be extracted from the Euclidean time-dependence of a suitable two-point correlation function. The contribution of the corresponding state can be disentangled from the rest by inserting the sources at large enough time-distances. Very often, however, the statistical error of the Monte Carlo estimate grows exponentially with the distance, and in practice it is not possible to find a window where statistical and systematic errors are both under control $[1,2]$. A widely used strategy to mitigate this problem is to reduce the systematic error by constructing interpolating operators with a reduced overlap over the excited states $[3,4]$. The lowest energy is then extracted at very short time-distances by assuming a negligible contamination from excited states. This procedure is not entirely satisfactory from a conceptual and practical point of view. The exponential problem remains unsolved, and the sources are usually optimized so that the correlator shows a single exponential decay in the short time range allowed by the statistical noise. A solid evidence that a single state dominates the correlation function, i.e. a long exponential decay over many orders of magnitude, is thus missing.

Here we propose a computational strategy to solve the exponential problem. We rewrite the path integral as a sum of functional integrals each giving the contribution from the states with definite symmetry properties. The ratio of each integral over the one with vacuum quantum numbers is then computed with a hierarchical integration procedure $[5,6]$. By iterating over several levels, the path integral ratios and the correlation functions can be estimated with a cost growing asymptotically as a power of the time extent of the lattice.

In the long term the main goal of our programme is to apply this strategy to nonAbelian gauge theories. However the latter are rather complicated quantum theories with many degrees of freedom. We find it appropriate to explain and check the basic ideas behind our strategy in a considerable simpler environment, one dimensional quantum systems with a non trivial parity symmetry. The generalization to quantum mechanical systems with many degrees of freedom and more involved symmetries is quite straightforward, while an accompanying paper is dedicated to describe the implementation in four dimensional Yang-Mills theories [7]. We test the strategy explicitly by simulating a one dimensional harmonic oscillator. The cost of the simulations scales as expected. In particular the statistical error on the two-point correlation function is exponentially reduced with respect to the standard Monte Carlo procedure, and at large time distances it is lowered by many orders of magnitude. 


\section{Path integral in quantum mechanics}

We are interested in a generic quantum mechanical system with one degree of freedom. Comprehensive reviews on this subject can be found in many text books, and therefore we concentrate only on those properties which are relevant to this paper. The time evolution of the system is described in the Heisenberg representation by constant states and time-dependent operators. The dynamics is fixed by the Hamiltonian operator

$$
\hat{H}=\frac{\hat{p}^{2}}{2 m}+V(\hat{x})
$$

where $V(\hat{x})$ is an external potential, $\hat{x}$ and $\hat{p}$ are the position and momentum operators whose commutator is $[\hat{x}, \hat{p}]=i$. We define a parity transformation $\hat{\mathcal{P}}$ as

$$
\hat{\mathcal{P}} \hat{x}=-\hat{x} \hat{\mathcal{P}}, \quad \hat{\mathcal{P}}^{2}=\mathbb{1},
$$

and we focus on Hamiltonian operators which are parity-invariant, i.e. $[\hat{\mathcal{P}}, \hat{H}]=0$. The energy eigenstates $\left|E_{i}^{s}\right\rangle$, with

$$
\hat{H}\left|E_{i}^{s}\right\rangle=E_{i}^{s}\left|E_{i}^{s}\right\rangle
$$

can be labeled by their parity $s= \pm$ and by the index $i=0,1, \ldots$ corresponding in each parity sector to the eigenvalue $E_{i}^{s}$ in ascending order. The dynamics of the system can be studied by introducing an Euclidean (imaginary) time lattice with $a$ being the spacing between consecutive time slices and $T=N a$ the time extent of the lattice. Eventually the discretization can be removed by extrapolating the results obtained at small lattice spacings to the "continuum" limit. The transfer operator between two consecutive time slices is defined as

$$
\hat{\mathcal{T}}=e^{-\frac{a}{2} V(\hat{x})} e^{-a \frac{\hat{p}^{2}}{2 m}} e^{-\frac{a}{2} V(\hat{x})}
$$

and its eigenvalues

$$
\hat{\mathcal{T}}\left|\mathcal{E}_{i}^{s}\right\rangle=e^{-a \mathcal{E}_{i}^{s}}\left|\mathcal{E}_{i}^{s}\right\rangle
$$

satisfy $\mathcal{E}_{i}^{s}=E_{i}^{s}+O\left(a^{2}\right)$ at small lattice spacing. The functional integral of the system is defined as

$$
Z \equiv\left(\frac{m}{2 \pi a}\right)^{-N / 2} \operatorname{Tr}\left[\hat{\mathcal{T}}^{N}\right]=\left(\frac{m}{2 \pi a}\right)^{-T / 2 a}\left[\sum_{i} e^{-\mathcal{E}_{i}^{+} T}+\sum_{i} e^{-\mathcal{E}_{i}^{-} T}\right]
$$

where Tr indicates the trace over all states in the Hilbert space of the theory. The time-ordered products of operators can be defined in the usual way. The two-point function of $\hat{x}$, for instance, is

$$
\left\langle x_{l} x_{k}\right\rangle \equiv \frac{1}{\operatorname{Tr}\left[\hat{\mathcal{T}}^{N}\right]} \operatorname{Tr}\left[\hat{\mathcal{T}}^{N-|l-k|} \hat{x} \hat{\mathcal{T}}^{|l-k|} \hat{x}\right]
$$


For large enough values of $N$ and $|l-k|$, it is dominated by a single contribution ${ }^{1}$

$$
\left\langle x_{l} x_{k}\right\rangle=2\left|\left\langle\mathcal{E}_{0}^{-}|\hat{x}| \mathcal{E}_{0}^{+}\right\rangle\right|^{2} e^{-\frac{T}{2}\left(\mathcal{E}_{0}^{-}-\mathcal{E}_{0}^{+}\right)} \cosh \left[\left(\mathcal{E}_{0}^{-}-\mathcal{E}_{0}^{+}\right)\left(\frac{T}{2}-a|l-k|\right)\right]+\cdots,
$$

from which $\left(\mathcal{E}_{0}^{-}-\mathcal{E}_{0}^{+}\right)$and $\left|\left\langle\mathcal{E}_{0}^{-}|\hat{x}| \mathcal{E}_{0}^{+}\right\rangle\right|$can be extracted. In the coordinate basis $|x\rangle$ defined by

$$
\hat{x}|x\rangle=x|x\rangle, \quad\left\langle x^{\prime} \mid x\right\rangle=\delta\left(x^{\prime}-x\right), \quad \hat{\mathbb{1}}=\int d x|x\rangle\langle x|,
$$

the momentum operator and its eigenvectors $|p\rangle$ are

$$
e^{-i \hat{p} \Delta}|x\rangle=|x+\Delta\rangle, \quad\langle x \mid p\rangle=\frac{1}{\sqrt{2 \pi}} e^{i p x},
$$

and the matrix elements of the transfer operator

$$
\left\langle x_{n+1}|\hat{\mathcal{T}}| x_{n}\right\rangle \equiv\left(\frac{m}{2 \pi a}\right)^{1 / 2} T_{n+1, n}
$$

can be computed explicitly

$$
T_{n+1, n}=e^{-a L_{n+1, n}}
$$

with

$$
L_{n+1, n} \equiv \mathcal{L}\left(x_{n+1}, x_{n}\right)=\frac{m}{2}\left(\frac{x_{n+1}-x_{n}}{a}\right)^{2}+\frac{V\left(x_{n+1}\right)}{2}+\frac{V\left(x_{n}\right)}{2},
$$

where we have adopted the conventions $x\left(t_{n}\right)=x_{n}$. By inserting $N$ times the identity in Eq. (2.6), the functional integral can be written as

$$
Z=\int \prod_{n=0}^{N-1}\left[d x_{n} T_{n+1, n}\right],
$$

where periodic boundary conditions $x_{0}=x_{N}$ are assumed. If we define the action of the theory to be

$$
S=a \sum_{n=0}^{N-1} L_{n+1, n},
$$

the functional integral and the two-point function can be rewritten as usual

$$
\begin{aligned}
Z & =\int \prod_{n=0}^{N-1} d x_{n} e^{-S} \\
\left\langle x_{l} x_{k}\right\rangle & =\frac{1}{Z} \int \prod_{n=0}^{N-1} d x_{n} e^{-S} x_{l} x_{k} .
\end{aligned}
$$

For $N$ large enough, the vacuum expectation values of time-ordered products correspond to the correlation functions of the statistical system defined by the action in Eq. (2.15).

\footnotetext{
${ }^{1}$ The ground state is assumed to be parity invariant.
} 


\section{Signal-to-noise ratio in path integral Monte Carlo}

Correlation functions can be computed with Monte Carlo techniques by averaging their values over a sample of configurations extracted with a probability $e^{-S} / Z[8]$. The statistical variance associated, for instance, to the two-point correlation function is

$$
\sigma^{2}=\left\langle x_{l}^{2} x_{k}^{2}\right\rangle-\left\langle x_{l} x_{k}\right\rangle^{2}
$$

and, at asymptotically large time separations $|k-l| \leq N / 2$, the signal-to-noise ratio can be easily computed in the underlying quantum field theory

$$
\frac{\left\langle x_{l} x_{k}\right\rangle}{\sigma}=\frac{\left|\left\langle\mathcal{E}_{0}^{-}|\hat{x}| \mathcal{E}_{0}^{+}\right\rangle\right|^{2}}{\left|\left\langle\mathcal{E}_{0}^{+}\left|\hat{x}^{2}\right| \mathcal{E}_{0}^{+}\right\rangle\right|} e^{-a\left(\mathcal{E}_{0}^{-}-\mathcal{E}_{0}^{+}\right)|l-k|}+\cdots
$$

The exponential decrease of this ratio with the time distance can be traced back to the fact that each configuration gives a contribution to the signal which decreases exponentially in time while contributes $O(1)$ to the noise at any time distance. This represents, up to date, one of the main limitations which prevents reliable estimates of energy levels via Monte Carlo simulations for many systems. It is particularly severe in presence of many degrees of freedom, as in lattice gauge theories, where the generation of configurations tends to be rather expensive $[1,2]$.

\section{Factorization of the functional integral}

The invariance of the system under parity transformations can be exploited to write the functional integral in a partly factorized form. If we define the parity eigenstates

$$
|x, \pm\rangle=\frac{1}{\sqrt{2}}(|x\rangle \pm|-x\rangle), \quad \hat{\mathcal{P}}|x, \pm\rangle= \pm|x, \pm\rangle
$$

the identity operator can be written as

$$
\hat{\mathbb{1}}=\frac{1}{2} \sum_{s= \pm} \int d x|x, s\rangle\langle s, x| \text {. }
$$

The invariance of the Hamiltonian under parity implies

$$
\left\langle s^{\prime}, x_{n+1}|\hat{\mathcal{T}}| x_{n}, s\right\rangle=\left(\frac{2 m}{\pi a}\right)^{1 / 2} T_{n+1, n}^{s} \delta_{s^{\prime} s}
$$

with

$$
\begin{aligned}
T_{n+1, n}^{s} & =\frac{1}{2} e^{-a \Lambda_{n+1, n}^{+}}\left\{e^{a \Lambda_{n+1, n}^{-}}+s e^{-a \Lambda_{n+1, n}^{-}}\right\} \\
\Lambda_{n+1, n}^{ \pm} & =\frac{1}{2}\left\{\mathcal{L}\left(-x_{n+1}, x_{n}\right) \pm \mathcal{L}\left(x_{n+1}, x_{n}\right)\right\}
\end{aligned}
$$


and the functional integral can be written as

$$
Z=\sum_{s= \pm} Z^{s}, \quad Z^{s}=\int \prod_{n=0}^{N-1} d x_{n} T_{n+1, n}^{s}
$$

Since the transfer matrix between singlet (parity even) states of the group transformations is real and positive if $e^{-a L_{n+1, n}}$ is so, it is appropriate to define

$$
T_{n+1, n}^{+} \equiv e^{-a L_{n+1, n}^{+}}=e^{-a \Lambda_{n+1, n}^{+}} \cosh \left\{a \Lambda_{n+1, n}^{-}\right\},
$$

and to cast the functional integrals in the form

$$
Z^{+}=\int \prod_{n=0}^{N-1} d x_{n} e^{-S^{+}}, \quad Z^{-}=\int \prod_{n=0}^{N-1} d x_{n} e^{-S^{+}} \prod_{m=0}^{N-1} \tanh \left\{a \Lambda_{m+1, m}^{-}\right\},
$$

where

$$
e^{-S^{+}} \equiv e^{-a \sum_{n=0}^{N-1} L_{n+1, n}^{+}}, \quad \frac{T_{n+1, n}^{-}}{T_{n+1, n}^{+}}=\tanh \left\{a \Lambda_{n+1, n}^{-}\right\}
$$

The path integral is thus rewritten as a sum of two functional integrals giving the contribution from parity even and odd states respectively. Each integrand is a product of transfer matrix elements between quantum states with definite parity. The ratio of the functional integrals in the two sectors can be written in the suggestive form

$$
\frac{Z^{-}}{Z^{+}}=\frac{1}{Z^{+}} \int \prod_{n=0}^{N-1} d x_{n} e^{-S^{+}} \prod_{m=0}^{N-1} \tanh \left\{a \Lambda_{m+1, m}^{-}\right\},
$$

and the two-point correlation function reads $(k<l)$

$$
\begin{aligned}
\left\langle x_{l} x_{k}\right\rangle & =\frac{1}{Z} \int \prod_{n=0}^{N-1} d x_{n} e^{-S^{+}}\left\{x_{l} \prod_{m=k}^{l-1} \tanh \left\{a \Lambda_{m+1, m}^{-}\right\} x_{k}\right. \\
& \left.+\prod_{m=l}^{N-1} \tanh \left\{a \Lambda_{m+1, m}^{-}\right\} x_{l} x_{k} \prod_{m=0}^{k-1} \tanh \left\{a \Lambda_{m+1, m}^{-}\right\}\right\} .
\end{aligned}
$$

\subsection{Hierarchical integration formula}

To proceed further we introduce the notion of time-slice of variable thickness [6]. It is the sub-lattice of all lattice points with time coordinate in a given interval $[k, l]$ with the boundaries being the points at times $k$ and $l$. The theory on a thick time-slice can be studied independently of the surrounding lattice if the variables at the boundaries are kept fixed, a decoupling property which is a consequence of the locality of the action density in time $[5,6]$. For any given function $\mathcal{O}$ of the variables $x_{n}$ belonging to the sub-lattice only, we can define the average

$$
[\mathcal{O}]_{l, k}^{+} \equiv \frac{1}{Z_{l, k}^{+}} \int \prod_{n=k+1}^{l-1} d x_{n} e^{-S_{\text {sub }}^{+}} \mathcal{O}
$$


where

$$
Z_{l, k}^{+}=\int \prod_{n=k+1}^{l-1} d x_{n} e^{-S_{\mathrm{sub}}^{+}},
$$

and the subscript "sub" indicates that the action is restricted to the sub-lattice. The average $[\mathcal{O}]_{l, k}^{+}$is a well defined function of $x_{k}$ and $x_{l}$ only, and does not depend on the $x_{n}$ elsewhere on the lattice. This implies that for a multi-local function $\mathcal{O}=\mathcal{O}_{1} \mathcal{O}_{2}$, where $\mathcal{O}_{1}$ and $\mathcal{O}_{2}$ depend on coordinates in non-overlapping lattice thick-slices, the recursive relation

$$
\left[O_{1} O_{2}\right]_{l, k}^{+}=\left[\left[O_{1}\right]_{l, m}\left[O_{2}\right]_{m, k}\right]_{l, k}^{+}
$$

holds. An instructive example is given by the ratio of the parity odd over the parity even partition functions which can be written as

$$
\frac{Z_{l, k}^{-}}{Z_{l, k}^{+}}=\left[\prod_{n=k}^{l-1} \tanh \left\{a \Lambda_{n+1, n}^{-}\right\}\right]_{l, k}^{+} .
$$

Thanks to the recursive relation in Eq. (4.14), the ratio

$$
\mathcal{R}_{l, k} \equiv\left\{\begin{array}{ll}
\frac{Z_{l, k}^{-}}{Z_{l, k}^{+}} & l>k+1 \\
\frac{T_{k+1, k}^{-}}{T_{k+1, k}^{+}} & l=k+1 \\
1 & l=k
\end{array},\right.
$$

with $\mathcal{R}_{l, k}=\mathcal{R}_{k, l}$ for $l<k$, satisfies

$$
\mathcal{R}_{l, k}=\left[\mathcal{R}_{l, m} \mathcal{R}_{m, k}\right]_{l, k}^{+} .
$$

The two-point correlation can thus be written as

$$
\left\langle x_{l} x_{k}\right\rangle=\frac{1}{Z} \int \prod_{n=0}^{N-1} d x_{n} e^{-S^{+}}\left\{x_{l} \mathcal{R}_{l, k} x_{k}+\mathcal{R}_{N, l} x_{l} x_{k} \mathcal{R}_{k, 0}\right\} .
$$

Similar expressions can be obtained for other correlation functions with insertions of parity odd operators. The Eqs. (4.12) and (4.14) are the main building blocks of the computational strategy followed in the next sections.

\section{Multilevel simulation algorithm}

The transfer matrix representation

$$
\mathcal{R}_{l, k}=\frac{\left\langle-, x_{l}\left|\mathcal{T}^{|l-k|}\right| x_{k},-\right\rangle}{\left\langle+, x_{l}\left|\mathcal{T}^{|l-k|}\right| x_{k},+\right\rangle}
$$




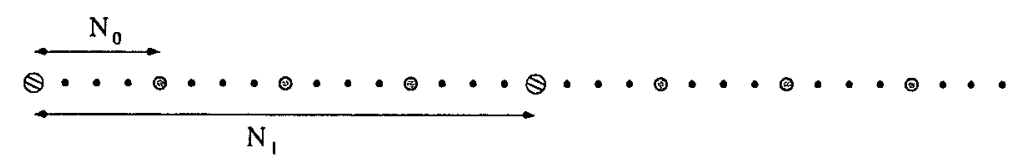

Figure 1: Three-level hierarchical structure of the lattice with blocks of length $N_{0}, N_{1}$, and $N_{2}=N$.

implies that the ratio $\mathcal{R}_{l, k}$ decreases exponentially in time for large enough distances $|l-k|<N / 2$, i.e. $\mathcal{R}_{l, k} \propto \exp \left\{-a\left(\mathcal{E}_{0}^{-}-\mathcal{E}_{0}^{+}\right)|l-k|\right\}$. In a Monte Carlo computation which extracts configurations with a probability distribution $e^{-S^{+}} / Z^{+}$, the contribution from each configuration is of the same order as the expected signal for any value of the time distance $|l-k|$. Therefore the exponential decrease of the signal-to-noise ratio described in section 3 does not apply. The numerical computation, however, is cheaper only if the effort for computing $\mathcal{R}_{l, k}$ does not grow exponentially with the time distance. This can be achieved by introducing a multi-level simulation algorithm $[5,6]$ which implements a stochastic hierarchical integration based on Eq. (4.14). In the following we are interested in computing the ratio of path integrals $Z^{-} / Z_{+}$for several values of $T$, and the two-point correlation $\left\langle x_{l} x_{k}\right\rangle$ at any distance $|l-k|$ on a given lattice. As an example, we describe in some detail a three-level procedure, the generalization to more levels being straightforward.

We start by splitting a lattice of length $N$ in a three-level hierarchical structure of non-overlapping blocks of length $\left(N_{0}, N_{1}, N_{2}\right)$ with $N_{2}=N$, see Fig. 1. The computation of the two-point correlation function is carried out in the following steps:

1. Generate a thermalized configuration of $x_{n}$ by spanning the full lattice with several sweeps of a Metropolis update algorithm.

2. Compute an estimate of the thick-slice integrals

$$
\begin{array}{cc}
{\left[x_{l} \prod_{n=k}^{l-1} \tanh \left\{a \Lambda_{n+1, n}^{-}\right\}\right]_{k+N_{0}, k}^{+},} & {\left[x_{m} \prod_{n=m}^{k+N_{0}-1} \tanh \left\{a \Lambda_{n+1, n}^{-}\right\}\right]_{k+N_{0}, k}^{+},} \\
{\left[\prod_{n=k}^{k+N_{0}-1} \tanh \left\{a \Lambda_{n+1, n}^{-}\right\}\right]_{k+N_{0}, k}^{+},} & {\left[x_{m} x_{l} \prod_{n=m}^{l-1} \tanh \left\{a \Lambda_{n+1, n}^{-}\right\}\right]_{k+N_{0}, k}^{+},} \\
{\left[x_{l} x_{m} \prod_{n=k}^{m-1} \tanh \left\{a \Lambda_{n+1, n}^{-}\right\}\right.} & \left.\prod_{n=l}^{k+N_{0}-1} \tanh \left\{a \Lambda_{n+1, n}^{-}\right\}\right]_{k+N_{0}, k}^{+}, \quad l, m \in\left(k, N_{0}+k\right),
\end{array}
$$

in each block of length $N_{0}$ (level 0 ) by keeping fixed the boundaries. The estimates are obtained numerically by averaging over $n_{0}$ configurations generated by spanning the interior of each block with the Metropolis update algorithm. 
3. Assemble the thick-slice integrals computed in step 2 to build up the analogous observables in the blocks of length $N_{1}$ (level 1). The corresponding thick-slice integrals are estimated by repeating this procedure over $n_{1}$ configurations generated by spanning each block of level 1 with the Metropolis update algorithm.

4. Repeat the same as in step 3 for the level 2 by averaging over $n_{2}$ configurations.

5. Repeat $n_{\text {conf }}$ times the steps from 1 to 4 .

The algorithm produces always exact results, up to statistical errors of order $n_{\text {conf }}^{-1 / 2}$, provided that $n_{0}, n_{1}$, and $n_{2}$ are $\geq 1$. Its efficiency, however, strongly depends on the values of these parameters which have to be tuned case by case.

\subsection{Scaling of the algorithm}

For a given relative statistical error on the ratio $Z^{-} / Z^{+}$, the cost of the algorithm scales with a power of the time extent of the lattice. To show this, let us assume that we have computed the ratio with a $n$-level algorithm. The total cost $C(N)$ of the simulation can thus be written as

$$
\frac{C(N)}{C\left(N_{0}\right)}=e^{\Sigma_{i} \log \left(c_{i}\right)}
$$

where $c_{i}$ is the cost of computing the ratio at the level $i$ starting from the results at the level $i-1$, and it is proportional to the number of configurations $n_{i}$ generated at the $i^{\text {th }}$ level. Since $n \propto \log \left(N / N_{0}\right)$, the cost $C(N)$ grows as a power of $\left(N / N_{0}\right)$. As a concrete example we can consider the case $N_{i} / N_{i-1}=r$ and $c_{i}=c$, which yields

$$
\frac{C(N)}{C\left(N_{0}\right)}=\left(\frac{N}{N_{0}}\right)^{\log (c) / \log (r)} .
$$

If we further assume the ansatz $c=\alpha r^{\beta} e^{\gamma \cdot r}$ then

$$
\frac{C(N)}{C\left(N_{0}\right)}=\alpha^{n-1}\left(\frac{N}{N_{0}}\right)^{\beta+\gamma \cdot r / \log (r)} .
$$

For $\gamma>0$ the multi-level integration algorithm achieves an exponential reduction of the cost of the simulation, whereas for $\gamma=0$ it changes the overall factor from $\alpha$ to $\alpha^{n-1}$. One expects the algorithm to start scaling with $\gamma=0$ when the time-extent $N_{i}$ is large enough for the partition function to be dominated by a single exponential.

\section{Numerical tests}

We test our strategy with a linear harmonic oscillator defined by the potential

$$
V(x)=\frac{1}{2} m \omega^{2} x^{2} .
$$



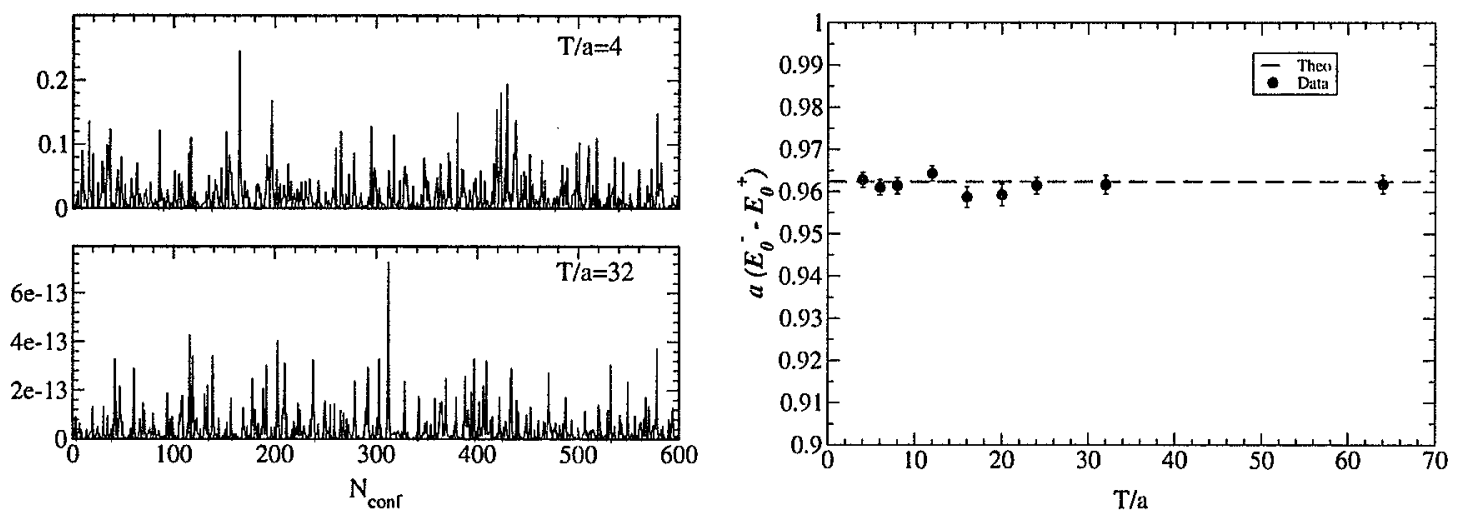

Figure 2: Left: history plot for $N=4,32$. Right: effective energy difference as defined in Eq. (6.5) versus $T / a$.

This system has a non-trivial parity symmetry, and it simple enough for eigenvalues and eigenvectors of the transfer operator to be computed exactly on the lattice [9]. If we define

$$
\bar{\omega}^{2}=\omega^{2}\left(1+\frac{a^{2} \omega^{2}}{4}\right), \quad a \tilde{\omega}=\log \left[1+a \bar{\omega}+\frac{a^{2} \omega^{2}}{2}\right]
$$

the transfer matrix eigenvalues $e^{-a \mathcal{E}_{i}^{s}}$ are given by

$$
\mathcal{E}_{n}^{+}=\tilde{\omega}\left(2 n+\frac{1}{2}\right), \quad \mathcal{E}_{n}^{-}=\tilde{\omega}\left(2 n+\frac{3}{2}\right), \quad n=0,1, \ldots
$$

and the matrix element we are interested in is

$$
\left\langle\mathcal{E}_{0}^{-}|\hat{x}| \mathcal{E}_{0}^{+}\right\rangle=\frac{1}{\sqrt{2 m \bar{\omega}}} .
$$

We have simulated harmonic oscillators for several values of am, aw, and $N$ chosen within reasonable ranges. Here we show the results for $a m=1, a \omega=1$ and several values of $N$, so that they can be directly compared with Ref. [9]. The performance of the algorithm and the results for the other parameter choices are in line with expectations.

The algorithm described in the previous section has a tree-like structure, and at each level the following parameters need to be specified: the thickness $N_{i}$ of the associated time-slices, the number of measurements $n_{i}$ for computing the time-slice average, and the number of time-slice updates $n_{i}^{\text {up }}$ between measurements. We fix their values first at the lowest level, then at the next-to-lowest one, and so on. At each level the values of $n_{i}$ and $n_{i}^{\mathrm{up}}$ are determined by requiring that the typical fluctuations of $\mathcal{R}_{l, k}$ due to the variation of the boundaries coordinates $x_{k}$ and $x_{l}$ dominate with respect to the statistical uncertainty on the Monte Carlo estimate. Depending on the value of $N$, we 

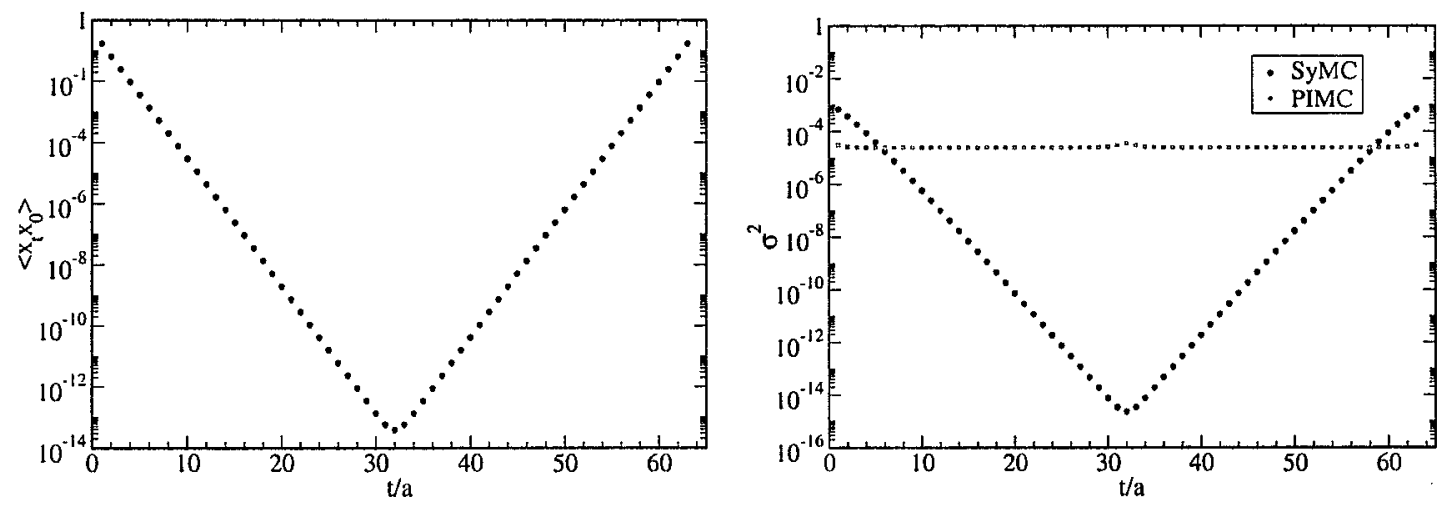

Figure 3: Left: two-point correlation function versus the time distance $t / a$ of the sources. Right: errors on the correlation function as obtained with the multi-level algorithm (SyMC) and with the standard path integral Monte Carlo (PIMC).

fix the thickness of the lowest level ${ }^{2}$ to $N_{0}=3,4$. For the higher levels $N_{i}$ is always 3-5 times the thickness of the previous one.

We have computed the ratio $Z^{-} / Z^{+}$for several values of $N$ in the range $N=4$ 64. For each run the number of configurations and the other parameters are chosen so that the cost of the simulations scales roughly proportionally to $N$. In the left plot of Figure 2 we show the Monte Carlo history for the first 600 configurations generated with $N=4$ and $N=32$. Similar plots are obtained for the other values of $N$ considered. The fluctuations of the data are very regular, and a standard statistical analysis is fully justified. The results for the effective energy difference

$$
\tilde{\omega}_{\text {eff }}(T)=-\frac{1}{T} \log \left(\frac{Z^{-}}{Z^{+}}\right)
$$

are shown in the right plot of Figure 2 together with the theoretical prediction. As expected, the statistical error is roughly constant over the whole range of values of $N$. The presence of a plateau signals that the ratio of multiplicities of the lowest states in the two sectors is 1 . The level of the plateau is in perfect agreement with the theoretical expectation of $a \tilde{\omega}=0.9624$ given in Eq. (6.2).

In the left plot of Figure 3 we show the two-point correlation function computed on a lattice with $N=64$ points, with statistical errors being smaller than symbols. It is estimated with a three-level algorithm with $\left(N_{0}, n_{0}, n_{0}^{\mathrm{up}}\right)=(4,48,3),\left(N_{1}, n_{1}, n_{1}^{\mathrm{up}}\right)=$ $(16,48,3),\left(N_{2}, n_{2}, n_{2}^{\text {up }}\right)=(64,48,3)$ and $n_{\text {conf }}=150$. At each level 2 hits per update were enough to have an acceptance of roughly $75 \%$. The estimate is very precise at all

\footnotetext{
${ }^{2}$ The peculiar regularity in the spectral properties of the harmonic oscillator makes the signal-tonoise ratio of the variable $\tanh \left\{a \Lambda_{m+1, m}^{-}\right\}$of $O(1)$. The cost of a standard integration scheme without multi-level already scales with a power-law, and it is therefore a viable option for this system. We do not expect this to happen in presence of many degrees of freedom, such as quantum field theories, and we find instructive to concentrate on the multi-level algorithm also here.
} 

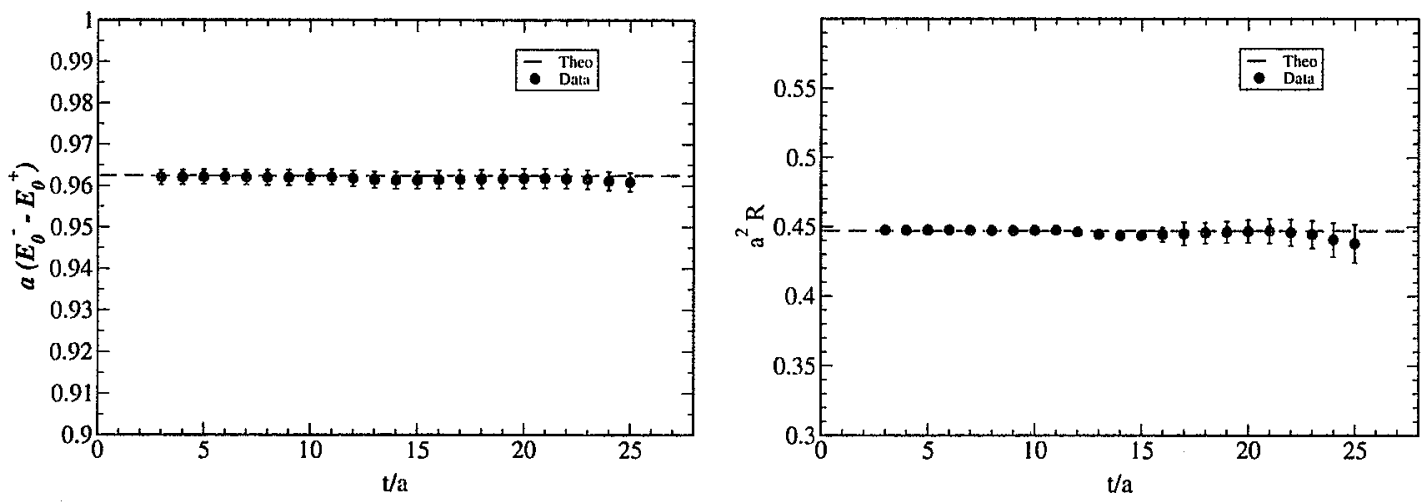

Figure 4: Left: effective energy difference extracted by solving Eq. (2.8) at any timedistance $t / a$. Right: the ratio $R$ defined as in Eq. (6.6)

time distances. The error is shown on the right plot of the same Figure. The signal-tonoise ratio is depleted, as expected, (only) inversely proportional to the time distance of the sources. For comparison in the same plot it is also shown the statistical error obtained with a standard Monte Carlo procedure which needed roughly the same CPU time. It is clear that with our strategy the statistical error is exponentially reduced, and at large time distances it is lowered by many orders of magnitude. The effective

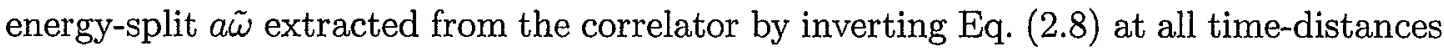
is shown in the left plot of Figure 4, and it is in perfect agreement with the theoretical expectation. On the right plot of the same figure it is shown the ratio

$$
R=\frac{\left\langle x_{l} x_{k}\right\rangle e^{\tilde{\omega} \frac{T}{2}}}{2 \cosh \left[\tilde{\omega}\left(\frac{T}{2}-a|l-k|\right)\right]},
$$

which agrees very well with the analytical result $R=(2 m \bar{\omega})^{-1}=0.4472 \ldots$ given in Eq. (6.4).

\section{Conclusions}

In this paper we have proposed a strategy to compute the contribution to the path integral from the subset of states with definite quantum numbers. We have checked the correctness and effectiveness of our strategy with an explicit simulation of a one dimensional harmonic oscillator. Our numerical experiments confirm that the cost for computing the energy of the first parity odd state grows linearly with the time extent of the lattice. At a fixed numerical effort the statistical error on the two-point correlation function is exponentially reduced with respect to the standard Monte Carlo procedure, and at large time distances it is lowered by many orders of magnitude. Our strategy is general enough to be applied to quantum systems with more degrees of freedom and 
more involved symmetry groups. A first application to non-Abelian Yang-Mills theories on the lattice is reported in Ref. [7].

\section{Acknowledgments}

We thank Martin Lüscher for many stimulating discussions and for the constant encouragement.

\section{References}

[1] G. Parisi, Phys. Rept. 103 (1984) 203.

[2] G.P. Lepage, TASI 89 Summer School, Boulder, CO, Jun 4-30, 1989.

[3] M. Albanese et al., Phys. Lett. B192 (1987) 163.

[4] M. Teper, Phys. Lett. B183 (1987) 345.

[5] G. Parisi, R. Petronzio and F. Rapuano, Phys. Lett. B128 (1983) 418.

[6] M. Lüscher and P. Weisz, JHEP 09 (2001) 010, hep-lat/0108014.

[7] M. Della Morte and L. Giusti, in preparation.

[8] N. Metropolis et al., J. Chem. Phys. 21 (1953) 1087.

[9] M. Creutz and B. Freedman, Ann. Phys. 132 (1981) 427. 
